\title{
Post-Critical Care COVID-19 Patient Benefits from a Robotic Patient-Guided Suspension System for Pulmonary Rehabilitation
}

\section{Dear Editor,}

The first case of coronavirus disease 2019 (COVID-19) in Singapore was diagnosed on 23 January 2020. As the number of COVID-19 cases increased in Singapore, ${ }^{1}$ the healthcare system has had to manage more critical care patients and the sequelae of such prolonged, severe illness. In the rehabilitation of post-critical care COVID-19 patients, aspects of both pulmonary as well as neuromuscular rehabilitation exist. It is interesting that our patient who experienced frequent exertional desaturation benefitted from the use of a robotic patient-guided suspension system for mobilisation.

Written informed consent was obtained from the patient. No approval was sought from Institutional Review Board as this was a case report. Our patient is a 61-year old Chinese male who was pre-morbidly well when he presented to his general practitioner with symptoms of fever and dry cough on 11 March 2020 (Day 2 of illness). He was treated with oral antibiotics but did not improve. He presented at our acute care hospital on Day 9 with a persistent cough and new-onset breathlessness. Chest radiograph revealed a left-sided pneumonia (Figure 1) and he was treated with meropenem, azithromycin, as well as oseltamivir. He deteriorated the next day and was intubated for acute respiratory distress syndrome (ARDS) complicated by type 1 respiratory failure. He tested positive for the SARS-CoV-2 virus by PCR testing, and was started on lopinavir/ritonavir (Kaletra) as well as interferon beta-1b. Due to side effects, treatment was switched to hydroxychloroquine. Six days of intravenous methylprednisolone for refractory wheeze was administered with clinical response. After mechanical ventilation for 15 days, he was extubated on Day 24 .

$\mathrm{He}$ was referred for inpatient rehabilitation on Day 30, after 2 negative COVID-19 swab results. $\mathrm{He}$ was oxygen-independent at rest but could only speak in phrases, and had exertional dyspnoea as well as resting tachycardia. Range of motion was full throughout and manual muscle testing via the

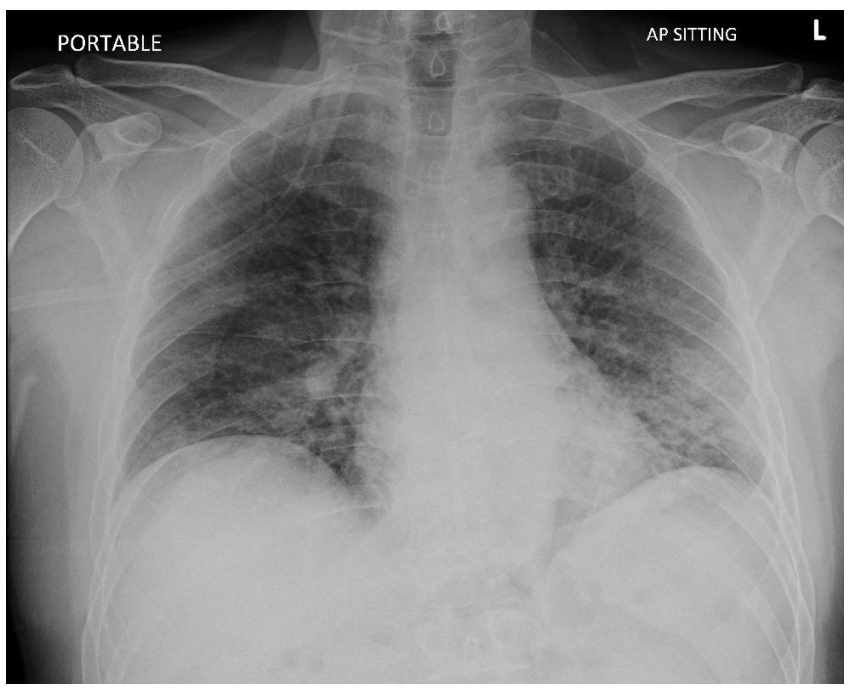

Figure 1: Patient's chest X-ray on admission

Medical Research Council muscle scale (MRC) demonstrated a power of 4 in all limbs, but with poor endurance, and muscular atrophy in the lower limbs was noted. Peripheral sensation was intact and he had no areflexia or hyporeflexia that would suggest critical illness polyneuropathy. The patient also had poor truncal control and dynamic standing balance. He fatigued easily on sit-to-stand tasks, required supervision for transfers, and could only walk $4 \mathrm{~m}$ with contact-assistance from therapists before he desaturated. The motor aspect of his Functional Independence Measure (Motor FIM) was scored as 59 (out of a total of 91 points).

He continued to have anxiety symptoms but no further passive suicidal ideations from his stay in critical care. He scored 4 on the anxiety component of the Hospital Anxiety and Depression Scale (HADS-A), and 6 on the depression component (HADS-D).

Upon transfer to our rehabilitation unit on Day 31, he was assessed by a multidisciplinary team. He continued daily conventional physiotherapy and occupational therapy as tolerated. A dietician reviewed him for $4 \mathrm{~kg}$ weight loss (from $72 \mathrm{~kg}$ ) during the 
illness and prescribed Ensure milk supplement as well as vitamins. Our patient had recurrent thoughts about his intensive care unit (ICU) stay, but did not exhibit symptoms of post-traumatic stress disorder (PTSD). He feared being ostracised upon discharge and his anxiety about breathlessness was exacerbated by fears of virus re-activation. A mental health therapist engaged him in relaxation techniques and cognitive reframing.

It is becoming known that cardiovascular involvement is common in patients with severe COVID-19, and cardiomyopathy resulting from myocarditis, profound systemic inflammation, or microvascular dysfunction is associated with a worse prognosis. ${ }^{2}$ As the patient had resting tachycardia and was planned for an increased intensity of rehabilitation, we organised cardiac enzymes and echocardiography to rule out cardiovascular disease - these were found to be normal. He then began training with the Andago V2.0 robot (Hocoma) which is a dynamic patient-guided suspension system for overground walking. Initial support requirements were $2.5 \mathrm{~kg}$ partial weights bilaterally (minimal weight support amounting to $5 \mathrm{~kg}$ in total) with $2 \mathrm{~L} / \mathrm{min}$ supplemental oxygen via intranasal cannula. He achieved a distance of $302 \mathrm{~m}$ on his first session which lasted half an hour. After 6 consecutive sessions of robotic gait training (also conducted through the weekend), he ambulated $368 \mathrm{~m}$, with $2.5 \mathrm{~kg}$ weight supports, without any further oxygen support (Figure 2).

The patient was discharged on Day 44 of his illness, after 13 days of inpatient rehabilitation. On discharge, he was walking independently without gait aids, and was independent in basic activities of daily living. His 6-minute walk test distance was $232 \mathrm{~m}$ and 10-meter walk test speed was $5.7 \mathrm{~s}$ with a gait speed of $1.87 \mathrm{~m} / \mathrm{s}$. HADS-A was reduced to 0 and HADS-D to 2. The patient achieved his goal of community ambulation.

\section{Discussion}

The patient presented to the acute care hospital on Day 9 of symptoms and was subsequently diagnosed with COVID-19 infection. In early March, testing was not as widely available and only selected patients with persistent symptoms were tested. ${ }^{3}$ Current statistics suggest that up to $6 \%$ of COVID19 -infected patients may require ICU stay and intubation. ${ }^{4}$ During the earlier phase of the outbreak in Singapore, the proportion of patients developing respiratory failure and needing mechanical ventilation was $15 \% .^{5}$ This patient deteriorated on Day 10 of illness as was similar to what has been described. ${ }^{6}$ Besides ARDS, patients could suffer from shock, myocardial dysfunction, and acute kidney injury. ${ }^{4}$ Central nervous system involvement includes dysgeusia, hyposmia, altered consciousness levels, and neuropsychological manifestations. Critical illness myopathy and neuropathy, as well as other neurological syndromes such as Guillain-Barré syndrome have also been reported. ${ }^{7}$ This could potentially amount to substantial disability and the rehabilitative needs in many severely-affected countries such as Italy have been shown to be great. ${ }^{7}$

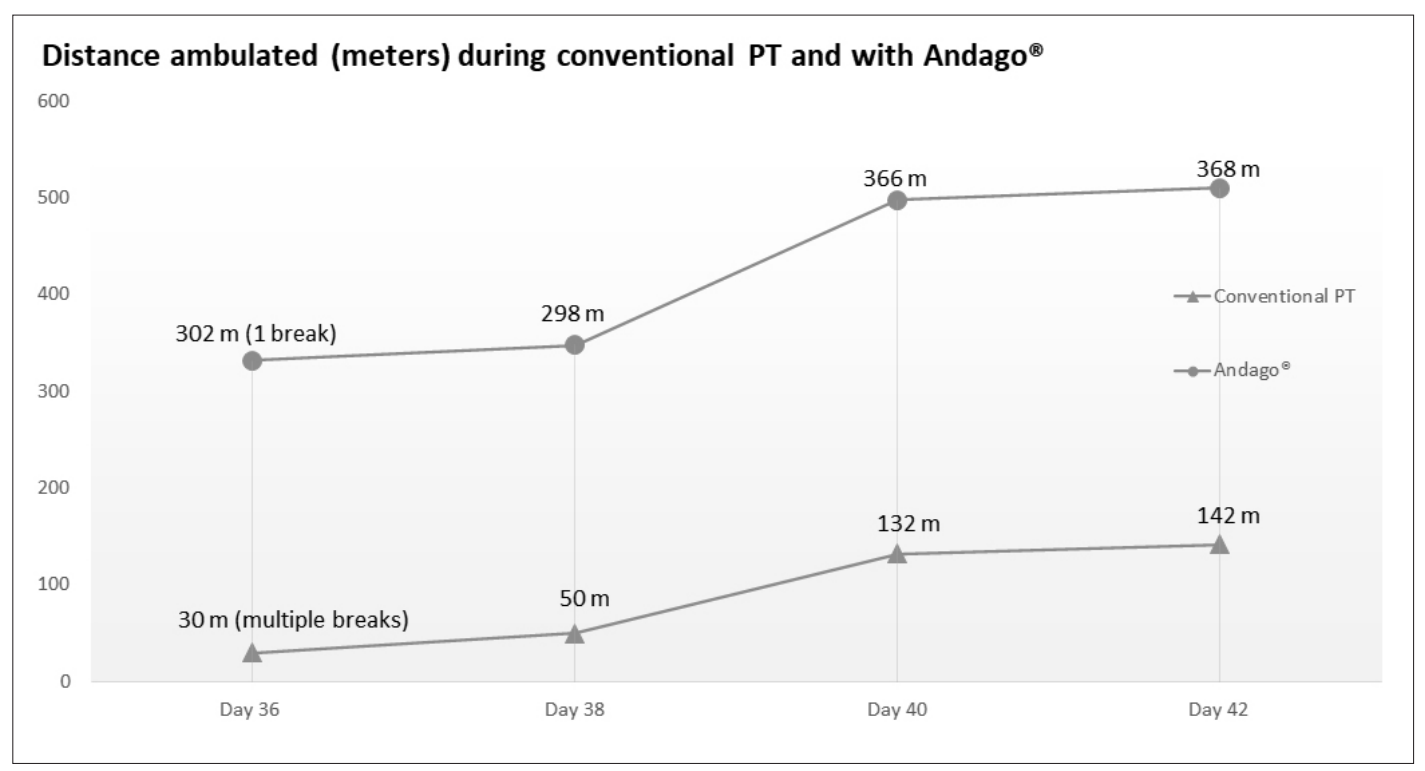

Figure 2: Comparison of distance ambulated using conventional physiotherapy and Andago 
The rehabilitation of a post-critical care patient with COVID-19 requires a multidisciplinary approach. Besides the 2 main areas that require rehabilitation, namely pulmonary and neuromuscular rehabilitation, ${ }^{8}$ other areas include nutrition and mental health. Of particular concern in these patients are the severe respiratory impairments that may preclude them from intensive therapies. ${ }^{9}$

Our patient desaturated early during activity and that impacted the intensity of the rehabilitation that he could tolerate. From our previous experience with Andago for another pulmonary rehabilitation patient, ${ }^{10}$ we applied this robotic therapy which provided partial weight support, taking away the need for $t$ herapists to support the patient. The Andago is a relatively-new robotic device that is classified in the category of patient-guided suspension systems ${ }^{11}$ (Figure 3). It has sensors and control algorithms to assist with propulsion and manoeuvring of the frame, adjustments to maintain patient stability, and also assists with weightbearing. Patients are not confined to a fixed area and are allowed to walk overground as well as explore the environment. For patients with a Functional Ambulatory Classification (FAC) of 2, usually only 1 therapist is needed to control the device and monitor the patient. In this first experience of applying Andago for a post-critical care COVID-19 patient, the physician was present during the first session to ensure that it was suitable and that the patient could tolerate the treatment. During the session, with minimal supplemental oxygen $(1 \mathrm{~L} / \mathrm{min})$, the patient was able to ambulate 10 times the distance covered during conventional therapy. Many pulmonary rehabilitation patients experience a vicious cycle of early desaturation, limited mobility, and further deconditioning, limiting them to low rehabilitation intensities. We believe that the partial weight support helped the patient to reduce the work of carrying his body forward during ambulation. In our case, robotic therapy enabled our patient to walk much further than he otherwise could with conventional therapy, breaking out of the vicious cycle and building endurance, physical balance, as well as confidence. Achieving an unaided distance of $142 \mathrm{~m}$ at discharge meant that he was able to walk to a car, facilitating community mobility and further independent reconditioning.

The Andago robot was preferred to other available devices such as treadmills with ceiling hoists and end-effector robots for 3 main reasons. Firstly, the patient is able to select his gait speed and is able to stop walking whenever he needs a break. Secondly, there is close proximity to the therapist as compared

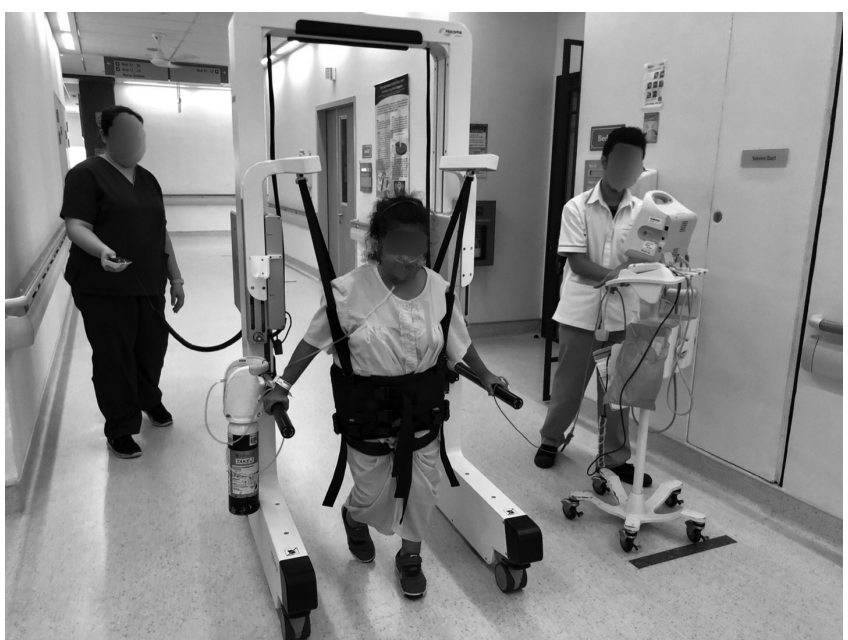

Figure 3: A patient using Andago ${ }^{\circledR}$ for pulmonary rehabilitation

to treadmills and end-effector devices, which allowed the patient to sit down on a chair to rest immediately after the harness was easily unclipped from the suspension system, without having to take any additional steps should he get breathless. Thirdly, the patient is not static and is allowed to explore the environment with Andago. This in itself was therapeutic to the patient who had been in isolation for weeks prior to transfer to inpatient rehabilitation. Andago has not been applied to COVID-19 patients from around the world based on available literature, and its use in pulmonary rehabilitation is a novelty in itself. ${ }^{10}$

This device may be beneficial in the pulmonary rehabilitation of patients who face early desaturation and dyspnoea post-severe infection. Post-infectious patients with COVID-19, H1N1, and other influenza-type pneumonias, as well as those with chronic restrictive lung disease may benefit from this intervention. ${ }^{10}$ To be suitable for this device, patients need to be ambulating with minimal-assistance (FAC 2), or demonstrate motor power of more than anti-gravity in the lower limbs (MRC >3). This device may be useful in dealing with a high volume of patients transferring out of ICU post-COVID-19 infection or in other future pandemics as it increases the intensity of rehabilitation while reducing the manpower and physical burden on therapists to support the patient during ambulation.

The Andago is also suitable for use as an adjunct in neurorehabilitation by allowing patients to achieve more repetitions of the gait cycle thereby increasing their functional gains ${ }^{12}$ (as illustrated similarly by our patient, Figure 2). 
We have also learnt that there is a need for better psychological support in patients recovering from severe COVID-19 infections due to both societal and self-perceived stigmata, as well as uncertainty over the disease course, especially in the earlier phase of the illness where they remain in isolation. In the recovery phase, symptoms of PTSD should be monitored. The involvement of mental health therapists in early phases of the illness, and the use of innovative approaches such as tele-rehabilitation for isolated patients, should likewise be considered. ${ }^{13}$

\section{Conclusion}

In view of the global pandemic of COVID-19, the healthcare system should be poised to manage more post-critical care patients, as well as the sequelae of prolonged severe illness. Our experience in treating this post-critical care patient with COVID-19 involved a multidisciplinary team. Innovative treatment approaches, such as robotic therapies that are traditionally used more commonly for neurological impairments arising from stroke and spinal cord injuries, may prove beneficial in the pulmonary rehabilitation of post-critical care COVID-19 patients as well. More evidence may be gathered as more patients utilise this device.

\section{REFERENCES}

1. Ministry of Health, Singapore. 57 more cases discharged; 1,106 new cases of COVID-19 infection confirmed. Available at: https://www.moh.gov.sg/news-highlights/details/57-more-casesdischarged-1-016-new-cases-of-covid-19-infection-confirmed. Accessed on 23 April 2020.

2. Guo T, Fan Y, Chen M, Wu X, Zhang L, He T, et al. Cardiovascular implications of fatal outcomes of patients with coronavirus disease 2019 (COVID-19). JAMA Cardiol 2020: e201017; DOI: 10.1001/ jamacardio.2020.1017

3. Hsu LY, Chia PY, Lim JF. The novel coronavirus (SARS-CoV-2) epidemic. Ann Acad Med Singapore 2020;49(3):105-7.

4. Phua J, Weng L, Ling L, Egi M, Lim CM, Divatia JV, et al. Intensive care management of coronavirus disease 2019 (COVID-19): challenges and recommendations. Lancet Respir Med 2020; 8:506-17. Erratum in: Lancet Respir Med 2020;8:e42

5. Ministry of Health, Singapore. Updates on Covid-19 (coronavirus disease 2019) local situation. Available at: https://www.moh.gov.sg/ covid-19. Accessed on 10 March 2020.

6. Goh KJ, Choong MC, Cheong EH, Kalimuddin S, Duu Wen S, Phua GC, et al. Rapid progression to acute respiratory distress syndrome: Review of current understanding of critical illness from COVID-19 infection. Ann Acad Med Singapore 2020; 49:108-18.

7. Brugliera L, Spina A, Castellazzi P, Cimino P, Tettamanti A, Houdayer E, et al. Rehabilitation of COVID-19 patients. J Rehabil Med 2020;52:jrm00046.

8. Zhao HM, Xie YX, Wang C. Recommendations for respiratory rehabilitation in adults with COVID-19. Chin Med J (Engl) 2020; DOI:10.1097/CM9.0000000000000848

9. McNeary L, Maltser S, Verduzco-Gutierrez M. Navigating coronavirus disease 2019 (Covid-19) in Physiatry: A CAN report for inpatient rehabilitation facilities. PM R 2020;12:512-5.

10. Tay SS, Alejandro C, Visperas EMW, Zainul Abideen A, Mohd Noor I. Success story of a case of arthrogyposis multiplex congenita with restrictive lung disease who underwent pulmonary rehabilitation using a robotic patient-guided suspension system. Proceedings of Singapore Healthcare 2020;29:67-9.

11. Esquenazi A, Talaty M. Robotics for lower limb rehabilitation. Phys Med Rehabil Clin N Am 2019;30:385-97.

12. French B, Thomas LH, Coupe J, et al. Repetitive task training for improving functional ability after stroke. Cochrane Database Syst Rev 2016;11:CD006073.

13. Simpson R, Robinson L. Rehabilitation after critical illness in people with COVID-19 infection. Am J Phys Med Rehabil 2020; 99:470-4.

San San Tay, ${ }^{1}$ MBBS (S'pore), MRCP (UK), MMed (Int Med, S'pore), Edmund JR Neo, ${ }^{2}$ MBBS (London), MRCP (UK), MMed (Int Med, S'pore), Mark MJ Tan, ${ }^{3} M D$ (S' pore), B.Eng. (CBE),

Pei Ling Tan, ${ }^{4} M D$ (S'pore), MRCP (UK), FAMS

${ }^{1}$ Department of Rehabilitation Medicine, Changi General Hospital, Singapore ${ }^{2}$ Rehabilitation Medicine, SingHealth Residency, Singapore

${ }^{3}$ Department of Rehabilitation Medicine, Changi General Hospital, Singapore

${ }^{4}$ Department of Rehabilitation Medicine, Changi General Hospital, Singapore

Address for Correspondence: Dr Tay San San, Department of Rehabilitation Medicine, Changi General Hospital, 2 Simei Street 3, Singapore 529889 Email: tay.san.san@singhealth.com.sg 